\title{
Direktausbildung Psychotherapie: Ein Plädoyer für eine offene Diskussion und für besonnenes Handeln
}

\author{
Winfried Rief \\ Klinische Psychologie und Psychotherapie, Psychotherapie-Ambulanz, Philipps-Universität Marburg, Deutschland
}

Das vorliegende Heft der Zeitschrift VerhaltenstherapiE nimmt sich im Besonderen des Themas Direktausbildung an. In der Pro-und-Contra-Rubrik werden von entsprechenden Fachvertretern pointiert die unterschiedlichen Blickwinkel auf das Thema dargestellt. Nachfolgend sollen im Editorial jedoch auch einige allgemeine Gedanken zu diesem Thema formuliert werden.

Das Psychotherapeutengesetz (PsychThG), in dem vor allem der Zugang nichtärztlicher Professionen zur beruflichen Tätigkeit als Psychotherapeuten geregelt ist, hat Revisionsbedarf. Dieser entsteht zum einen aus der Neufassung der Studiengänge durch den Bologna-Prozess, der bisher im Gesetz noch nicht berücksichtig ist. Andererseits legen aber auch diverse Konstruktionsfehler eine Revision nahe. Laut Position des Bundesministeriums für Gesundheit (BMG) sind die im PsychThG geschaffenen Heilberufe in ihrer Ausbildungsform zu weit entfernt von anderen akademischen Heilberufen wie der Ausbildung in der Medizin oder Zahnmedizin. Des Weiteren ist der ungleiche Zugang für die Psychologische Psychotherapie sowie die Kinder- und Jugendpsychotherapie nicht sinnvoll oder begründbar, insbesondere da er nicht nur unterschiedliche Studieninhalte, sondern unterschiedliche akademische Abschlussniveaus betrifft. Schließlich wird auch das PsychThG oftmals als Begründung herangezogen, um Psychotherapeuten in Ausbildung nur unter finanziell prekären Rahmenbedingungen anzustellen. Das BMG hat in dieser Situation darauf hingewiesen, dass eine substantielle Revision geplant ist, und lässt an verschiedenen Stellen erkennen, dass der Wunsch des BMG eine «Direktausbildung» in Psychotherapie wäre.

Bedauerlicherweise führte dies zu einer zwischenzeitlich stark emotionalisierten Diskussion. Dies ist verständlich, da viele unterschiedliche Interessensgruppen befürchten, dass ihre Interessen bei einer Revision zu kurz kommen könnten. Allerdings ist diese Emotionalisierung des Begriffes «Di- rektausbildung» auch wenig förderlich für eine ernsthafte Auseinandersetzung mit dem Thema. Für eine konstruktive Diskussion sollte man weniger Extremvarianten von Direktausbildungen als mögliches Teufelswerk an die Wand malen, sondern sachlich diskutieren, wie einerseits die bewährten Ausbildungsinhalte und -strukturen erhalten bleiben können, andererseits jedoch gegebenenfalls der oben beschriebene Revisionsbedarf des PsychThG berücksichtigt werden könnte.

\section{Erhalt bewährter Ausbildungselemente}

Bei jeder Revision besteht die Gefahr, dass, wie der Volksmund sagt, «das Kind mit dem Bade ausgeschüttet wird». Bei der bisherigen Psychotherapie-Ausbildung wurde von verschiedenen Expertengruppen festgestellt, dass diese $\mathrm{zu}$ einem qualitativ hochwertigen Berufsabschluss führt. Bei $85 \%$ erfolgt der Zugang über ein universitäres PsychologieStudium, und auch die nachfolgenden Elemente der Psychotherapie-Ausbildung wie z.B. die darin integrierten Workshops zur theoretischen Ausbildung, die Anleitung zum therapeutischen Handeln unter Supervision, die klinische Erfahrung (z.B. in Psychiatrien), die Selbsterfahrung und anderes wurden von den Ausbildungskandidaten sowie von der durch das BMG eingesetzten Gutachtergruppe inhaltlich als positiv und qualitativ als hochwertig beurteilt. Sicherlich ist es wichtig, dass Ausbildungscurricula eine gewisse Flexibilität zulassen, um sich an wissenschaftlichen Weiterentwicklungen anpassen zu können. In diesem Sinne kann diskutiert werden, ob an der einen oder anderen Stelle z.B. Neuentwicklungen aus den Neurowissenschaften oder Inhalte aus der Medizin/Pharmakologie stärker integriert werden sollten. Man beachte hierbei jedoch auch den starken Einzug dieser Inhalte in die Grundlagenfächer Biologische

\section{KARGER}

Fax +497614520714

Information@Karger.de

www.karger.com
(C) 2012 S. Karger GmbH, Freiburg

$1016-6262 / 12 / 0221-0004 \$ 38.00 / 0$

Accessible online at:

www.karger.com/ver
Prof. Dr. Winfried Rief

Klinische Psychologie und Psychotherapie, Psychotherapie-Ambulanz Philipps-Universität Marburg

Gutenbergstraße 18, 35032 Marburg, Deutschland

Tel. +49 6421 282-3641, Fax -8604

rief@staff.uni-marburg.de 
Psychologie, Emotionspsychologie, Allgemeine Psychologie. Auch sollte gegebenenfalls stärker eine «Lehre am Patienten» erfolgen, wobei manche universitäre Ausbildungsgänge bereits jetzt Fallseminare und ähnliche Veranstaltungen anbieten, die in diese Richtung gehen.

Auf der anderen Seite muss jedoch bei inhaltlichen Ergänzungen und Veränderungen grundsätzlich auch festgelegt werden, auf welche bisherigen Inhalte dann auch verzichtet werden soll. Möchte man Psychotherapeuten haben, denen die Grundlagen der Psychologie und damit die psychologischen Grundbegriffe zur Beschreibung der Phänomene von Patienten und Therapieprozessen nicht bekannt sind oder die keine Kenntnisse von intermittierender Verstärkung oder zum Extinktionslernen haben, obwohl dies für alle Psychotherapierichtungen real ablaufende Prozesse sind? Sollen sozialpsychologische, emotions- oder motivationspsychologische Aspekte gestrichen werden? Bei genauer Betrachtung muss man schlussfolgern, dass die psychologischen Grundlagenfächer eine Rolle haben, wie sie z.B. die Anatomie (als Grundlagenfach) für die Chirurgie (als Anwendungsfach) hat - die psychologischen Grundkonzepte werden definiert, die für eine gezielte Intervention und für ein wissenschaftlichprofundes Problemverständnis notwendig sind.

Aber auch das grundlegende wissenschaftliche Verständnis sowie die wissenschaftliche Methodenkompetenz werden bisher sehr geschätzt und heben sich positiv von anderen Ausbildungsgängen $\mathrm{ab}$, die mit einer weniger profunden Vermittlung wissenschaftlich-methodischer Aspekte einhergehen. Wird Psychotherapie als universitär zu vermittelnde Behandlungsform definiert, ist damit die Zielsetzung verbunden, dass Psychotherapeuten auch wissenschaftliche Weiterentwicklungen in ihrem Feld verstehen und adaptieren können, selbst wenn die Ausbildung abgeschlossen ist. Moderne Psychotherapieforschung $\mathrm{zu}$ verstehen setzt voraus, dass dieses profunde methodische Wissen vorliegt. Deshalb würde eine Reduktion in der Vermittlung wissenschaftlich-methodischer Grundkompetenz zunehmend Psychotherapie als universitäre und postuniversitäre Disziplin in Frage stellen und gegebenenfalls zu einem reinen Anwendungsfach degradieren. Eine substantielle Reduktion der Methodenausbildung bedrohe somit den akademischen Status von Psychotherapeuten.

Dass schließlich und endlich profunde Kenntnisse und Hintergründe zu diagnostischen Prozessen, zur Störungslehre sowie zu Interventionen notwendig sind, ist unbestritten. Gleiches gilt für die Inhalte der bisherigen postgradualen Psychotherapie-Ausbildung. Wenn für wichtige Themen wie Essstörungsbehandlung, Behandlung von Zwangserkrankten oder ähnlichem nur ein Wochenend-Workshop vorgesehen ist, ist dies sicherlich nicht überdimensioniert. Auch die praktische Ausbildung unter Supervision oder die Notwendigkeit der Praxiserfahrung z. B. in psychiatrischen Einrichtungen mag zwar bezüglich des Umfangs diskutiert werden, wird in ihrer Sinnhaftigkeit jedoch in der Regel bejaht. Diese wenigen Beispiele machen bereits deutlich, dass es schwierig werden dürfte, Ausbildungsinhalte sowohl aus dem Studium als auch aus der Psychotherapie-Ausbildung zu definieren, auf die man problemlos verzichten könnte, um sie durch andere Inhalte zu ersetzen.

\section{Was bedeutet im Kontext der Direktausbildung «Approbation"?}

Mit dem Begriff «Direktausbildung» ist erst einmal nur gemeint, dass am Ende des Studiums bereits die Möglichkeit zu einem Staatsexamen mit der Erteilung der Approbation möglich ist. Dies bedeutet nicht, dass dies das Ende der gesamten Aus- und Weiterbildungsstruktur darstellen soll oder mit einem in irgendeiner Form qualitativ befriedigenden Ausbildungsniveau verbunden ist. Juristisch gesehen bedeutet Approbation die Erteilung der Arbeitserlaubnis im Bereich der Heilkunde. Sie setzt voraus, dass Störungswissen bekannt ist und die positiven Möglichkeiten der therapeutischen Intervention, jedoch auch die möglichen Gefahren bewusst sind. Dies bedeutet nicht, dass die entsprechenden Therapien wirklich kompetent durchgeführt werden könnten. Vergleicht man dies wieder mit der Medizin, so definiert die Approbation die Einstiegsqualifikation z.B. des Arztes, der auf einer Assistenzarztstelle in der Psychiatrie anfängt, oder des Assistenzarztes, der in der Chirurgie seine Facharztqualifikation erwerben möchte. Die Approbation öffnet somit das Tor, um sich die praktische Erfahrung und Kompetenz anzueignen und zu vertiefen. Im bisherigen Bereich der Psychotherapie ist Approbation jedoch mit der Endqualifikation verbunden, wenn sozusagen die Vertiefung schon stattgefunden hat und auch ausreichend praktische Kompetenz erworben wurde. Hier besteht somit eine Diskrepanz zwischen dem Approbationsbegriff in der Medizin versus dem der bisherigen Psychotherapeutenausbildung.

\section{Wie könnte eine Direktausbildung aussehen?}

Nachfolgend soll durch einige Beispiele aufgezeigt werden, wie unterschiedlich die Konzepte sein können, die unter dem Stichwort «Direktausbildung» diskutiert werden.

\section{a) Radikales Modell Direktausbildung}

Es könnte ein komplett neuer Studiengang «Psychotherapie» kreiert werden, dessen Curriculum neu zusammengestellt wird. Dies hat einerseits eine gewisse Faszination der Freiheit der Ausgestaltung, jedoch andererseits auch die Problematik, ohne profunde Vorerfahrungen mit dem Gesamtcurriculum und der Qualität dieser neu kreierten Ausbildung in das Abenteuer des neuen Ausbildungswegs zu gehen. Bei neuen Fächern ist dies notwendig und sinnvoll. Allerdings haben wir im Bereich Psychotherapie jahrzehntelange Erfahrungen mit der Aus- und Weiterbildung, und es sind viele Strukturen ent- 
Tab. 1. Geforderte Ausbildungsinhalte laut Beschluss des 16. Deutschen Psychotherapeutentags und Überarbeitung durch den Vorstand der Bundespsychotherapeutenkammer (Oktober 2010; verkürzte Fassung)

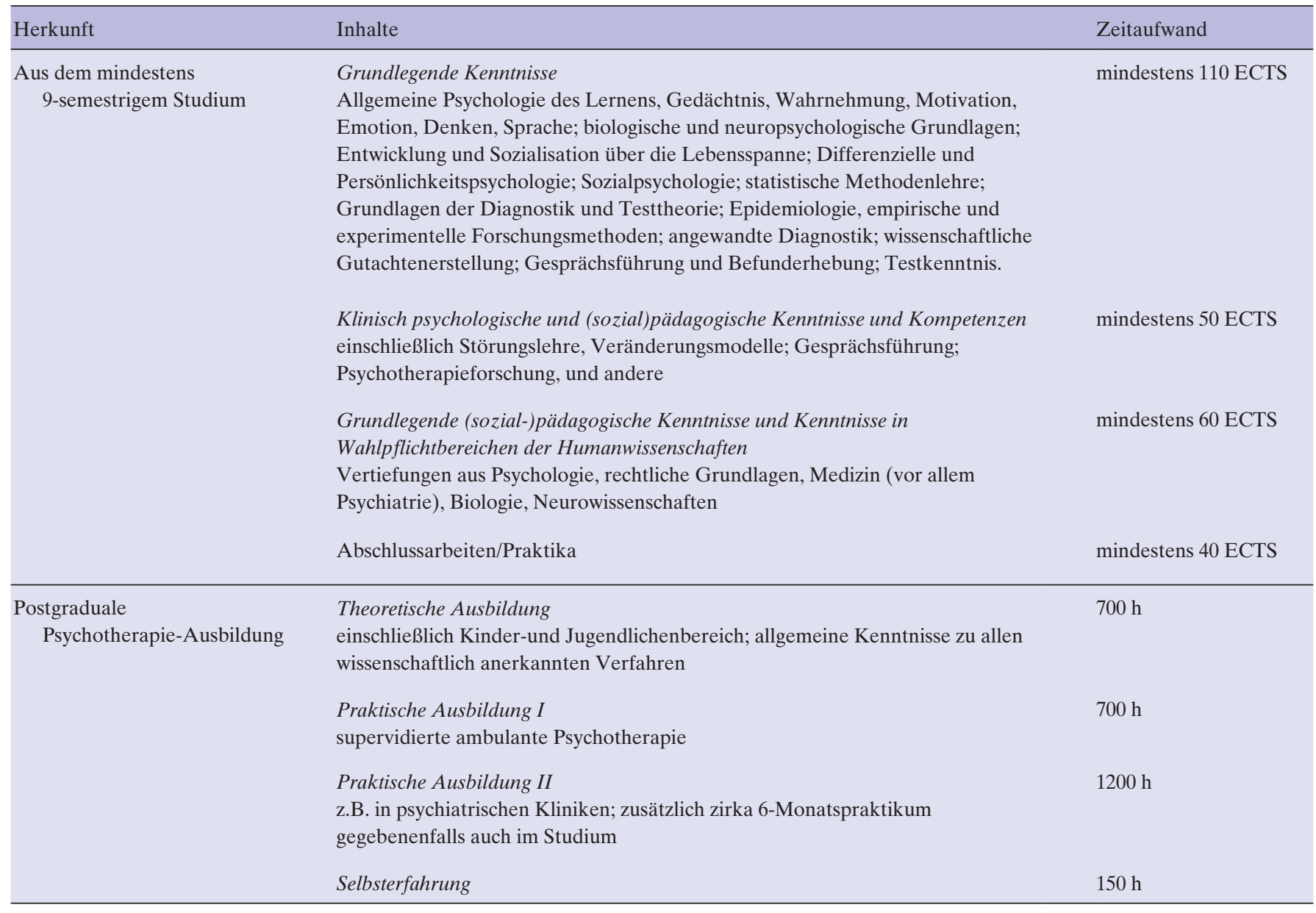

standen, die sinnvoll sind und zu einer qualitativ hochwertigen Ausbildung beitrugen. Deshalb stellt sich die Frage, ob der risikoreiche Weg einer radikalen Umstrukturierung wirklich Sinn macht. Nebenbei sind damit auch zahlreiche Zusatzfragen verknüpft: Wie soll die inhaltliche Überlappung zum bisherigen Psychologiestudium geregelt werden, wie werden universitäre Institute neu aufgeteilt, wie wird der Numerus clausus/Zugang zu diesem Studienfach geregelt, welche Finanzierungsmodelle stellen die Länder langfristig zur Verfügung, wenn die Einführungsanreize aufgebraucht sind usw. Wenn dies der Hauptzugangsweg für nichtärztliche Psychotherapeuten werden sollte, müssten pro Jahr mindestens 1500-2000 Studierende in solche Studiengänge aufgenommen werden, und insgesamt würden zirka 8000 Studienplätze benötigt. Wer bezahlt diese Studienplätze zu Lasten von wem? Neben der Konsentuierung der Studieninhalte wirft ein solches Modell somit zahlreiche administrative Fragen auf.

\section{b) Revisionsvorschlag der Bundespsychotherapeutenkammer} mit Ergänzung «Modellausbildungsgang Direktausbildung» Der Vorschlag der Bundespsychotherapeutenkammer sowie des 16. Deutschen Psychotherapeuten-Tages hat inhaltlich wesentliche Elemente der universitären und postgradualen Psychotherapie-Ausbildung definiert, über die hoher Konsens besteht (Tab. 1). Offensichtlich wurde es von der breiten Masse der Psychotherapeuten als notwendig angesehen, dass diese Inhalte vermittelt werden. Zusätzlich wird eine nur geringe Revision der postgradualen Psychotherapie-Ausbildung vorgeschlagen, so dass auch hier die meisten Elemente als «bewährt» gewertet wurden. Allerdings ermöglicht der Vorschlag des Psychotherapeuten-Tages auch Modellausbildungsgänge z.B. im Sinne einer Direktausbildung, die hoch kompatibel mit diesem Rahmenvorschlag sind. So könnte durch ein klinisch und praktisch angereichertes PsychologieStudium eine Approbation im Anschluss an den MasterAbschluss ermöglicht werden, zusätzlich könnte man dann die postgraduale Aus- bzw. Weiterbildung etwas kondensieren. Vereinfacht gesprochen würde damit vor allem die Approbation etwas vorgezogen, so dass in der anschließenden Weiterbildung mehr Rechtssicherheit besteht. Allerdings würde die sozialrechtliche Anerkennung auf jeden Fall eine vollständige Weiterbildung in Psychotherapie fordern, die wie bisher entweder an privaten Ausbildungsinstituten oder hochschulnahen Ausbildungsinstituten erfolgen könnte. 


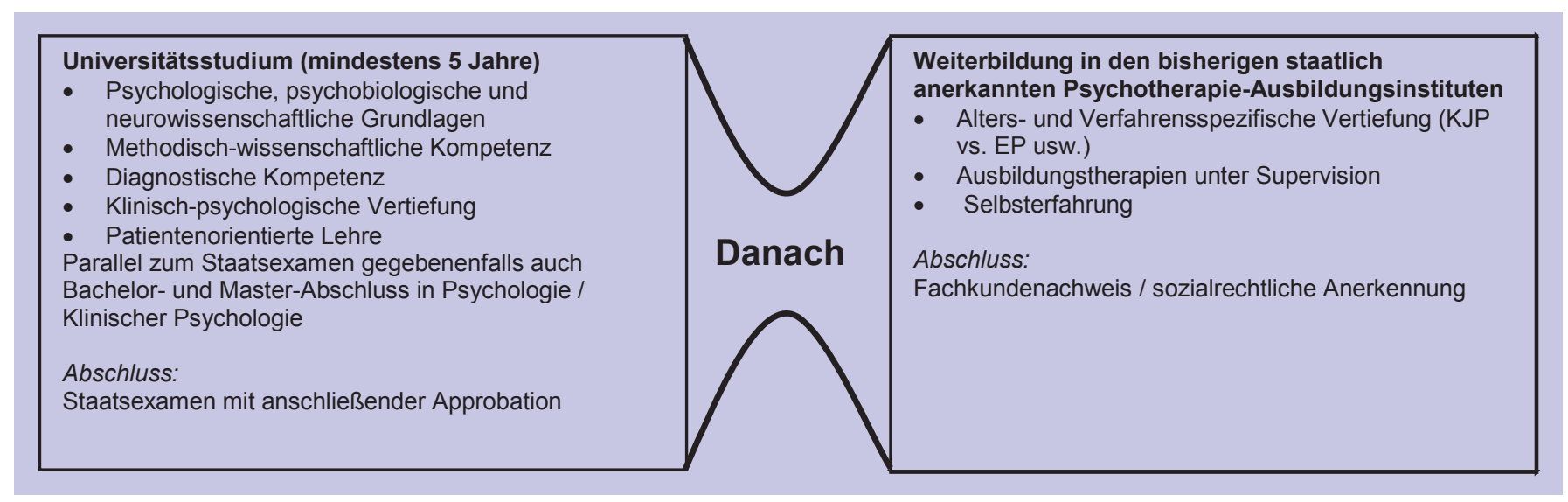

Abb. 1. Schematische Darstellung des Modells einer zweigliedrigen Aus- und Weiterbildung («moderates Modell einer Direktausbildung»).

\section{Moderates Modell Direktausbildung}

Während der Vorschlag b) hohe Flexibilität für verschiedene Ausbildungsgänge bietet, die einerseits völlig identisch zum bisherigen Ausbildungsgang sein könnten, jedoch auch Neuerungen ermöglichen, könnte ein moderates Modell Direktausbildung sozusagen als Untergruppe von Vorschlag b) verstanden werden. Die bisherigen Inhalte des für die meisten Psychotherapeuten zuführenden Psychologie-Studiums werden weiterhin gefordert, und auch die Psychotherapie-Ausbildung an privaten oder universitätsnahen Ausbildungsinstituten nach Abschluss des Psychologie-Studiums wird weiterhin als notwendig erachtet für die sozialrechtliche Anerkennung. Allerdings würde die Approbation/das Staatsexamen vorgezogen und somit zwischen Psychologie-Studium und Psychotherapie-Weiterbildung platziert (Abb. 1.).

\section{Plädoyer für eine nachhaltige Reform}

Damit eine aktuelle Revision des PsychThG auch eine nachhaltige und stabile Reform darstellt, ist es notwendig, dass die Ausbildungsqualität als solche behalten bleibt und die Patienteninteressen nach qualitativ-hochwertiger Behandlung im Vordergrund stehen. Auch müssen die verschiedenen Interessensgruppen, soweit sie berechtigte Interessen vertreten, so weit wie möglich berücksichtigt werden, um eine breite Unterstützung zu bekommen. Die zukünftige Regelung sollte aber auch eine gewisse Klarheit haben, so dass nicht zu viel Interpretationsspielraum besteht und sukzessive zunehmend «Schleichwege» ausprobiert werden, um an den Abschluss zu kommen. Es wird dafür plädiert, dass eine Revision deshalb mit einer gewissen Besonnenheit durchgeführt wird und keine Einladung zu abenteuerlichen Umbauprozessen erfolgt, die letztendlich zu einer sowohl qualitativ als auch administrativ schwer kalkulierbaren Spielwiese von Aus- und Weiterbildungen führen würden. Der Verzicht der Berücksichtigung bisheriger Ausbildungserfahrungen und bestehender Ausbildungsstrukturen kann nicht im Sinne einer qualitativ hochwertigen Heranführung an den Beruf sein. Sicherlich ist eine Angleichung an die Strukturen anderer Approbationsstudiengänge sinnvoll und erleichtert die juristische Einordnung; jedoch bedeutet dies auch hier keineswegs, dass alles komplett identisch sein muss. Bereits Medizin- und Zahnmedizin-Studium haben ihre Besonderheiten und Unterschiede, so dass hier ein gewisser Spielraum auch für die Psychotherapie bestehen dürfte. In Deutschland ist es gelungen, ein qualitativ hochwertiges Versorgungssystem Psychotherapie zu installieren. Dies sollte nicht allzu leichtfertig verspielt werden, auch wenn an der einen oder anderen Stelle Optimierungsbedarf ist. 